\title{
MORPHOLOGY AND OPTICAL PROPERTIES OF THIN SILICA FILMS CONTAINING BIMETALLIC Ag/Au NANOPARTICLES
}

\author{
G. R. Yashan, ${ }^{1}$ A. M. Eremenko, ${ }^{1}$ N. P. Smirnova, ${ }^{1}$ S. Suzer, ${ }^{2}$ \\ G. Ertas, ${ }^{2}$ and Ch. Tabor ${ }^{3}$ \\ UDC 544.77.051, 544.172.2, 661.682
}

\begin{abstract}
We have studied the optical spectra in the UV and visible regions, the morphology by scanning electron microscopy (SEM), and the X-ray photoelectron spectra (XPS) of bimetallic Ag/Au nanoparticles incorporated into transparent silicate films in the sol-gel transition stage. The bimetallic nanoparticles, obtained by a combination of photoreduction and thermal reduction, form structures of the alloy or core-shell type.
\end{abstract}

Key words: bimetallic silver and gold nanoparticles, silica films, photoreduction and thermal reduction, UV spectra, SEM, XPS.

Nanoparticles of noble metals, in particular gold and silver, have unique optical, electrical, and magnetic properties which are different from both the properties of the bulk metals and the properties of the individual atoms or ions. Synthesis and study of nanosized particles are of interest because of the possibility of using them in optoelectronics, chemical catalysis, as biological labels in diagnostics. Silica-based composites, modified by nanoparticles of noble metals, have stimulated considerable interest from the basic research and applied standpoint in connection with enhancement of catalytic, sensor, linear and nonlinear optical properties. The silica matrix (a dielectric) protects the nanoparticles from oxidation and an undesirable aggressive environment.

For certain purposes, use of bimetallic composites based on $\mathrm{Ag} / \mathrm{Au}$ is more effective than using nanocomposites containing only one type of nanoparticle. For example, in determining trace amounts of molecules, bimetallic nanoparticles display considerably more sensitivity in SERS analysis as biosensors than individual nanoparticles [1,2]. Ag and Au have the same lattice constants and are miscible in all proportions, forming alloys. The process of synthesizing nanoparticle $/ \mathrm{SiO}_{2}$ composites includes dissolving metal salts in silicon alkoxide, depositing these precursors onto glass substrates, and then calcinating in a different gaseous atmosphere. In this case, compaction of the matrix and reduction of the salts down to the corresponding metallic nanoparticles occur [3]. Pyrolytic synthesis of gold nanoparticles in sol-gel silica was done in [4]. Photodeposition of nanoparticles on the silica surface makes it possible to control the particle size [5]. The combination of UV irradiation and heat treatment facilitates formation of metallic nanoparticles from their salts in silica matrices, since reduction processes occur in both treatments. In [6], a new approach is suggested for synthesis of a bimetallic alloy from $\mathrm{Ag} / \mathrm{Au}$

${ }^{1}$ O. O. Chuiko Institute of Surface Chemistry, National Academy of Sciences of Ukraine, Vul. Generala Naumova, 17, Kyiv 03164, Ukraine. E-mail: annaerem@ukr.net.

${ }^{2}$ Department of Chemistry, Bilkent University, Ankara 06800, Turkey.

${ }^{3}$ Laser Dynamics Laboratory, School of Chemistry and Biochemistry, Georgia Institute of Technology, Atlanta, GA 30332-0400, USA.

Translated from Teoreticheskaya i Éksperimental'naya Khimiya, Vol. 44, No. 6, pp. 348-353, November-December, 2008. Original article submitted December 1, 2008. 


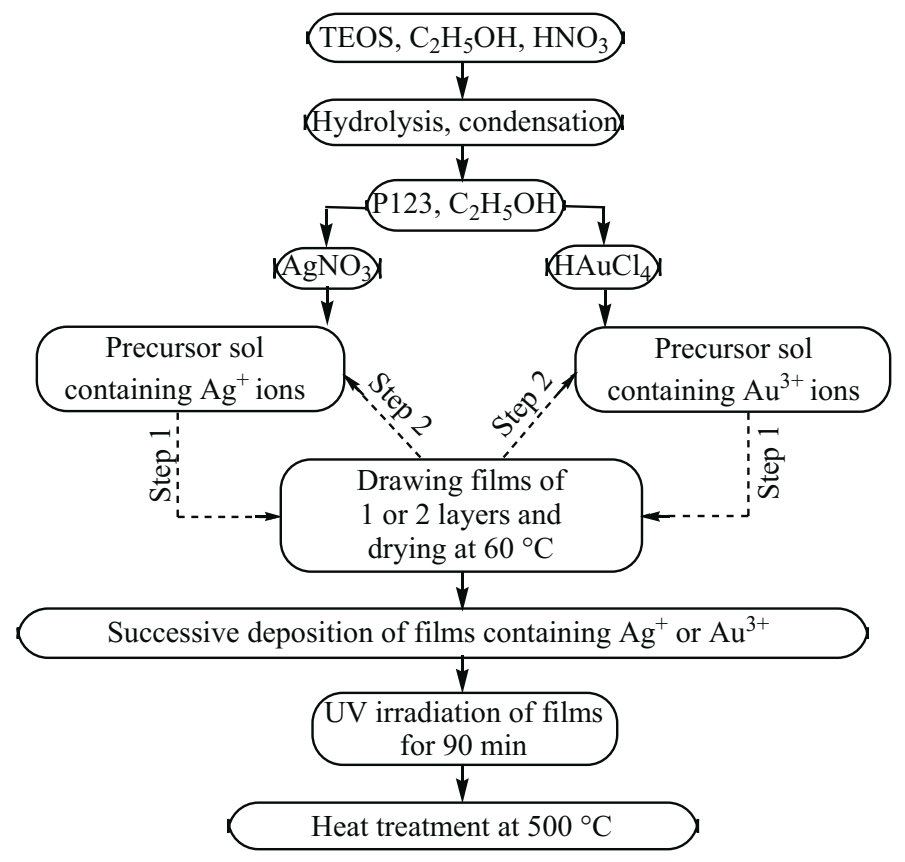

Fig. 1. Block diagram for obtaining the films.

nanoparticles in a silica film, including subsequent deposition of individual layers of the precursors $\mathrm{Ag}^{+} / \mathrm{SiO}_{2}$ and $\mathrm{Au}^{3+} / \mathrm{SiO}_{2}$ on glass substrates, followed by UV irradiation and heat treatment of the "bilayer" hydrogel films obtained. The authors utilized a complicated synthesis scheme, using TEOS mixed with glycidoxypropyl trimethoxysilane (GLYMO), aluminum acetylacetonate, and a mixture of alcohols. We can hypothesize that on heating, accompanied by hydrolysis of the silicon alkoxides and interdiffusion of the metal ions and particles, the interface between the films disappears and, as a result, continuous "monolayer" films are obtained that contain nanosized $\mathrm{Ag} / \mathrm{Au}$ alloys.

The aim of this work was to obtain Ag/Au bimetallic nanoparticles of different morphologies in porous silica sol-gel films, using UV irradiation and heat treatment as the routes for reduction of the corresponding ions down to the metals. The Ag and $\mathrm{Au}$ ions were introduced into the silica precursor both simultaneously and sequentially. In synthesis of the "bilayer" films containing one metal in the bottom layer of the film and the second metal in the top layer, we used the principle proposed in [6], considerably simplifying the synthesis procedure by using only TEOS as the $\mathrm{SiO}_{2}$ source and the template P123 to obtain a porous structure. We studied the optical spectra, used SEM to study the structure of the bimetallic nanoparticles, and measured the X-ray photoelectron spectra (XPS) of some of the composites obtained.

\section{EXPERIMENTAL}

The starting reagents $\mathrm{AgNO}_{3}, \mathrm{HAuCl}_{4}, 98 \%$ TEOS (Aldrich) were used without additional purification. The mesoporous silica films were obtained by the sol-gel method, by hydrolysis of the tetraethoxysilane using the triblock copolymer $\mathrm{P}_{123}(\mathrm{BASF})(\mathrm{PEO})_{20}(\mathrm{PPO})_{70}(\mathrm{PEO})_{20}$ as the template, similarly to what is presented in [7]. The gold and silver ions were introduced by different methods into the silica precursor in the sol-gel transition stage: both salts were simultaneously dissolved in the $\mathrm{SiO}_{2}$ sol and then the hydrogel was deposited on a glass substrate; the films of the hydrogel $\mathrm{Ag}^{+} / \mathrm{SiO}_{2}$ and $\mathrm{Au}^{3+} / \mathrm{SiO}_{2}$ were deposited successively on the substrate, forming "bilayer" silica films $\left(\mathrm{Ag} / \mathrm{SiO} \mathrm{O}_{2}: \mathrm{Au} / \mathrm{SiO}_{2}\right)$ with gold or silver clusters in the top layer. The films were obtained by thermal reduction or combined photoreduction and thermal reduction of the hydrogel silica films containing gold and silver ions. The films were exposed to UV radiation from a $1000 \mathrm{~W}$ mercury lamp for $90 \mathrm{~min}$, followed by heat treatment at $500^{\circ} \mathrm{C}$. 
Fig. 1 shows the block diagram for the sol-gel method for obtaining silica films containing bimetallic $\mathrm{Ag} / \mathrm{Au}$ nanoparticles. The alcohol molecules liberated during hydrolysis of silicon alkoxide play the role of a reducing agent for reducing the ions down to metallic nanoparticles on exposure to UV radiation. The hydrocarbon residues formed on annealing the films are reducing agents for the gold and silver ions during heat treatment.

Film 1 is a monolayer film $\mathrm{AgAu} / \mathrm{SiO}_{2}$ obtained by thermal reduction. $0.043 \mathrm{~g} \mathrm{AgNO}$ and $2 \mathrm{~mL}$ of an aqueous solution of $0.25 \mathrm{M} \mathrm{HAuCl}_{4}$ were added to the prepared precursor of film 1: a silica sol based on hydrolyzed TEOS in the presence of nitric acid, water, and the pore-former Pluronic 123 in ethyl alcohol (1TEOS : $0.16 \mathrm{HCl}: 39 \mathrm{EtOH}: 0.011 \mathrm{P}_{123}$ ). From the precursor obtained, containing both $\mathrm{Ag}^{+}$and $\mathrm{Au}^{3+}$ ions in $1: 1$ ratio, the film was deposited on a glass substrate by the dip-coating method. The film was slowly heated by a gradual rise in temperature up to $500^{\circ} \mathrm{C}$, and held at this temperature for $3 \mathrm{~h}$.

Film 2 is a monolayer film $\mathrm{AgAu} / \mathrm{SiO}_{2}$ with the same composition as film 1, obtained by a combination of photoreduction and thermal reduction. The hydrogel of the $\mathrm{AgAu} / \mathrm{SiO}_{2}$ film on a glass substrate was dried at $60{ }^{\circ} \mathrm{C}$, then exposed to UV radiation for $90 \mathrm{~min}$ and subjected to heat treatment at $500^{\circ} \mathrm{C}$. The combination of photoradiation treatment and heat treatment promotes formation of a larger number of bimetallic nanoparticles than the heat treatment method.

Films 3 and 4 are bilayer films. They are obtained by successive deposition on a glass substrate of the silica precursors, separately containing silver and gold ions. In film 3, the bottom layer contains silver ions $/ \mathrm{SiO}_{2}$, the top layer contains gold ions $/ \mathrm{SiO}_{2}$; in film 4, on the other hand, the bottom layer is gold in silica. Such films were heated at $60{ }^{\circ} \mathrm{C}$, then exposed to UV radiation from a $1000 \mathrm{~W}$ mercury lamp for $90 \mathrm{~min}$ and calcined with a gradual rise in temperature at $500{ }^{\circ} \mathrm{C}$ for $3 \mathrm{~h}$.

The metal content in all the films was $5 \mathrm{wt} . \% \mathrm{Ag}$ and $5 \mathrm{wt} . \% \mathrm{Au}$ relative to the silica.

\section{RESULTS AND DISCUSSION}

When exposed to UV radiation, gold and silver ions in an alcoholic solution form stable nanoparticle colloids. When an aqueous alcoholic solution of silver ions is exposed to UV radiation at a wavelength of $254 \mathrm{~nm}$, electron transfer occurs from the water or alcohol molecule to the silver ion in an instantaneous collision complex (the subscript "cage" means a solvent cage made up of water or alcohol molecules) [8]:

$$
\begin{gathered}
\left(\mathrm{Ag}^{+}, \mathrm{H}_{2} \mathrm{O}\right)_{\text {cage }} \stackrel{h v}{\longleftrightarrow}\left(\mathrm{Ag}, \mathrm{H}^{+}, \mathrm{OH}\right)_{\text {cage }}, \\
\left(\mathrm{Ag}, \mathrm{H}^{+}, \mathrm{OH}\right)_{\text {cage }} \rightarrow \mathrm{Ag}+\mathrm{H}^{+}+{ }^{\circ} \mathrm{OH}, \\
\left(\mathrm{Ag}^{+}, \mathrm{ROH}\right)_{\text {cage }} \stackrel{h v}{\longleftrightarrow} \mathrm{Ag}, \mathrm{H}^{+},\left(\mathrm{R}^{\prime} \mathrm{C} \cdot \mathrm{OH}\right)_{\text {cage }} .
\end{gathered}
$$

Ketyl radicals formed in the presence of alcohol (third line of the scheme) also reduce silver ions:

$$
\mathrm{Ag}^{+}+\mathrm{R}^{\prime} \mathrm{C} \cdot \mathrm{OH} \rightarrow \mathrm{Ag}+\mathrm{R}^{\prime} \mathrm{C}=\mathrm{O}+\mathrm{H}^{+}
$$

Processes of gold nanoparticle formation in the presence of alcohols when exposed to light with wavelength of both $254 \mathrm{~nm}$ and $365 \mathrm{~nm}$ occur in a complex of tetrachloroauric acid with alcohol, but are somewhat different from the processes for silver. After the appearance of the first portions of gold nanoparticles, catalytic (dark) reduction of gold ions occurs (when both ions are present in solution, the silver is also reduced on the surface of the gold nanoparticles). Furthermore, when gold ions are irradiated in an alcoholic medium, photogeneration of nanoparticles also proceeds by means of electron transfer within the $\mathrm{AuCl}_{4}^{-}$complex, from the chlorine ions to the gold ion. The reaction mechanism for intracomplex photoreduction is described in [9]. Thermal reduction of gold and silver ions occurs with participation of hydrocarbon radicals formed and liberated during heat treatment from alkoxide residues and alcohol molecules [10].

The absorption spectra of film 1 after heat treatment are shown in Fig. 2a (curve 1). The spectrum consists of two maxima, corresponding to surface plasmon resonance (SPR) bands of the gold $(565 \mathrm{~nm})$ and silver $(400 \mathrm{~nm})$ nanoparticles. We 

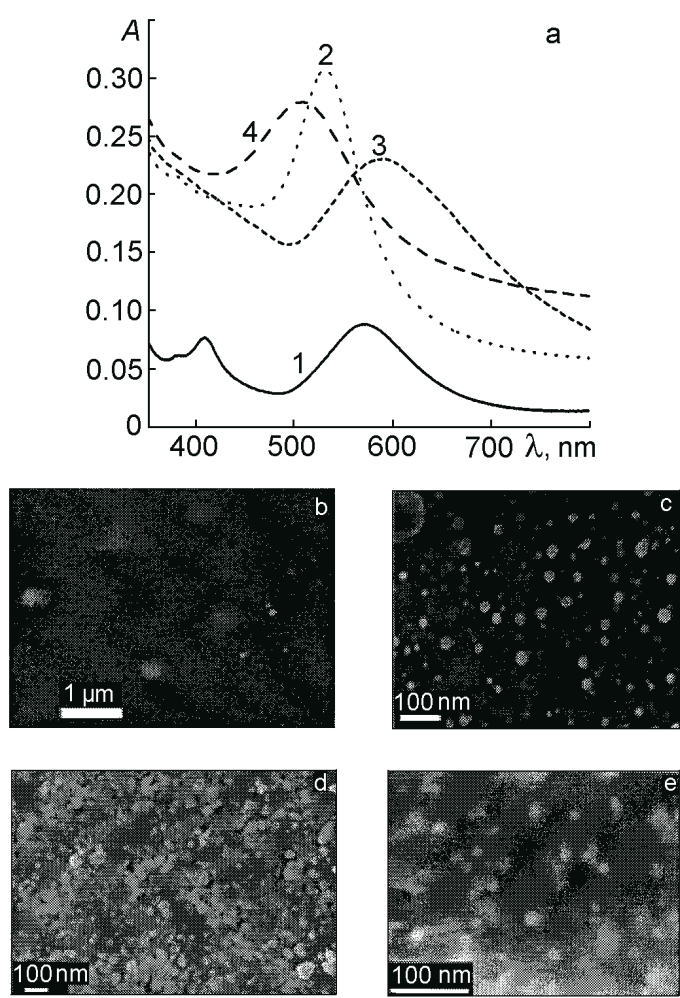

Fig. 2. Absorption spectra (a) and SEM micrographs of films 1 (b), 2 (c), 3 (d), and 4 (e).

know that decomposition of $\mathrm{HAuCl}_{4}$ occurs at $290^{\circ} \mathrm{C}$; at this temperature, nucleation and growth of the first gold clusters and nanoparticles occur, which melt when the temperature is raised further and form larger particles [11], the SPR bands for which therefore are somewhat shifted toward longer wavelengths. Formation of silver nanoparticles begins at $444{ }^{\circ} \mathrm{C}$; they form a separate phase and do not form an alloy with the gold nanoparticles. The existence of two types of nanoparticles is confirmed by micrographs obtained by scanning electron microscopy (SEM) (Fig. 2b). The gold nanoparticles have the shape of triangles and spheres; the silver nanoparticles have an irregular spherical shape. Thus upon thermal reduction of gold and silver ions found simultaneously in the precursor of a silica film, the gold and silver nanoparticles in the $\mathrm{SiO}_{2}$ matrix do not interact with each other and exist separately.

The absorption spectrum for film 2, exposed to UV radiation before heat treatment, contains an SPR band at $533 \mathrm{~nm}$ and a shoulder in the region of absorption by the silver nanoparticles (about $400 \mathrm{~nm}$ ) (Fig. 2a, curve 2). Preliminary UV irradiation of the silica film containing the metal ions, in the presence of alcohol molecules as a reducing agent, promotes simultaneous reduction of $\mathrm{AuCl}_{4}^{-}$and $\mathrm{Ag}^{+}$. SEM micrographs of this composite (Fig. 2c) and the SPR spectrum allow us to hypothesize that a $\mathrm{Ag}$ core/Au shell structure is formed, with enrichment of the gold shell by silver nanoparticles.

We also studied the effect of the sequence of deposition of the silica films with gold and silver ions ("bilayer" films) on their optical spectra and morphology. Fig. 2a (curve 3) and Fig. 2d show the characteristics of film 3, with a Ag-containing bottom layer and an Au-containing top layer, after UV irradiation and heat treatment. The main absorption maximum of the film at $587 \mathrm{~nm}$ is typical for SPR of gold; the shoulder at $400 \mathrm{~nm}$ corresponds to the SPR of silver nanoparticles. In the case of film 3, we also hypothesize formation of a Ag core/Au shell composite, where the gold shell is enriched with silver atoms, analogous to what is presented in [12]. In Fig. 2d, we show the SEM micrographs with bimetallic nanoparticles on the surface and in the pores of the silica film. Deviation of the ratio of the gold to silver nanoparticles from $1: 1$, obtained from calculation using the X-ray photoelectron spectra (Table 1), supports this hypothesis. The Ag: Au atomic ratio in this case is equal to $1.2: 1$. Table 1 gives the atomic ratios of the elements in the films, determined from XPS data. We should consider that the 
TABLE 1. Atomic Stoichiometry in the Surface

Layer of the Films

\begin{tabular}{c|c|c|c|c}
\hline \multirow{2}{*}{ Sample } & \multicolumn{5}{|c}{$\begin{array}{c}\text { Atomic ratios determined from XPS } \\
\text { measurements }\end{array}$} \\
\cline { 2 - 5 } & $\mathrm{Si}$ & $\mathrm{O}$ & $\mathrm{Au}$ & $\mathrm{Ag}$ \\
\hline \multirow{5}{*}{ Film 1 } & 1.0 & $2.2^{*}$ & 0.003 & 0.003 \\
Film 3 & 1.0 & 2.2 & 0.005 & 0.006 \\
Film 4 & 1.0 & 2.2 & 0.013 & 0.023
\end{tabular}

* Adsorbed water or surface - OH groups.

penetration depth of X-radiation in XPS is $10 \mathrm{~nm}$ and these tables refer to the surface layer of the film, which is about $100 \mathrm{~nm}$ thick.

The average particle radius in film 3, calculated from the SEM micrograph, is 10-20 nm and 40-60 nm.

In the spectrum of the bilayer film 4 (Fig. 2a, curve 4) with the bottom layer containing gold ions in the precursor silica film, after UV exposure and heat treatment at $500{ }^{\circ} \mathrm{C}$ the maximum of the SPR band is found at $500 \mathrm{~nm}$. In this case, we believe an alloy is formed between the gold and silver nanoparticles. Despite the fact that the position of the maximum does not correspond to the average position for the ratio $1 \mathrm{Au}$ : $1 \mathrm{Ag}$, i.e., about $470-480 \mathrm{~nm}$, the SPR band of film 4 is shifted compared with the absorption bands of the rest of the composites toward shorter wavelengths, in the region between the SPR bands of gold and silver. The silver nanoparticles are found in the top layer of the alloy, in which the predominant element is still gold [7]. The calculations of the particle size distribution, performed using the SEM data, show that for such an order of synthesis of the films, finer nanoparticles are formed, with an average radius from $5 \mathrm{~nm}$ to $30 \mathrm{~nm}$.

From the data given in Table 1, we see that when the metal ions are introduced simultaneously into film 1, after heat treatment the metal atoms are located in the surface layer of the film in $1: 1$ stoichiometry, while when there is a photochemical component in the reduction process (films 3 and 4), the shell of the bimetallic nanoparticle is enriched in silver atoms. In the case when silver is in the bottom layer and gold is in the top layer, the ratio is $\mathrm{Au}: \mathrm{Ag}=1: 1.2$; and when the order of film deposition is reversed, then the ratio is $1: 1.8$. Hence we can say that for films subjected to UV exposure and heat treatment, there is a tendency toward saturation of the top layer with silver nanoparticles.

Consideration of the interaction between the nanoparticles and bimetallic nanoparticles formed with the surface of the silica pores merits special attention. In principle, silica does not react with the gold and silver particles, but small particles within the pores are chemically reactive and react with the surrounding air molecules, in particular with oxygen, i.e., chemisorption of oxygen on the silver nanoparticles occurs, as is shown in [13]:

$$
\begin{gathered}
\mathrm{Ag}+\mathrm{O}_{2} \rightarrow \mathrm{Ag}^{+}+\mathrm{O}_{2 \text { ads }}^{-}, \\
4 \mathrm{Ag}+\mathrm{O}_{2} \rightarrow 4 \mathrm{Ag}^{+}+2 \mathrm{O}_{2 \text { ads }}^{2-} .
\end{gathered}
$$

The authors of [14] hypothesize that local porosity is formed at the nanoparticle-silica interface, where the metal nanoparticles are in contact with oxygen from the air. Gold nanoparticles about $10 \mathrm{~nm}$ in size also chemisorb oxygen [15]. Metal nanoparticles coated with a thin oxide film probably can undergo the reaction of electron transport from the metal to the surface. 
Thus formation of bimetallic nanoparticles is facilitated by combining UV irradiation and heat treatment of the sol-gel precursors of silica films that simultaneously contain two salts of the noble metals gold and silver. The composite films obtained, with an extended porous structure, are stable and transparent, and their absorption spectra contain the corresponding SPR bands for nanosized metallic composites. The morphology of the bimetallic Ag/Au nanoparticles obtained in silica sol-gel films in the form of an alloy, a core/shell structure, or separately existing gold and silver nanoparticles depends on the order in which the salts are introduced into the silica precursor and preliminary irradiation of the film before heat treatment. Porous sol-gel silicas containing bimetallic nanoparticles are of interest as potential biosensors and materials for surface-enhanced Raman spectroscopy.

This work was done within the joint program "Synthesis and properties of bimetallic (Ag, Au, Pt, Pd) nanoparticles for catalysis and antibacterial use" (project TBAG-U/179) of the National Academy of Sciences of Ukraine and the Scientific and Technological Research Council of Turkey (TUBITAK).

\section{REFERENCES}

1. Liu Yu-Chuan, Yu Chung-Chin, and Hsu Ting-Chu, Electrochem. Commun., 9, 639 (2007).

2. Xiangling Ren, Xianwei Meng, and Fangqiong Tang, Sensors Actuators B, 110, 358 (2005).

3. C. A. Morris, M. L. Anderson, R. M. Stroud, et al., Science, 284, 622 (1999).

4. H. Kozuka and S. Sakka, Chem. Mater., 5, 222 (1993).

5. F. Akbarian, B. Dunn, and J. Zink, J. Phys. Chem., 99, 3892 (1995).

6. S. Pal and G. De, Chem. Mater., 17, 6161 (2005).

7. G. Krylova, A. Eremenko, and N. Smirnova, Phys. Chem. Solids, 7, 50 (2006).

8. H. Hada, Y. Yonezawa, A. Yoshida, and A. Kurakake, J. Phys. Chem., 80, 2728 (1976).

9. S. Eustis, H.-Y. Hsu, and M. A. El-Sayed, J. Phys. Chem. B, 109, 235 (2005).

10. F. Kim, S. Connor, H. Song, et al., Chem. Int. Ed., 43, 3673 (2004).

11. S. Link and M. A. El-Sayed, J. Chem. Phys. B, 114, 2362 (2001).

12. Shiv Shankar S., Rai Akhilesh, and Ahmad Absar, J. Colloid Interface Sci., 275, 496 (2004).

13. W. Cai, Y. Zhang, J. Jia, and L. Zhang, Appl. Phys. Lett., 73, 2709 (1998).

14. W. Cai, H. Hofmeister, T. Rainer, and W. Chen, J. Nanoparticle Res., 3, 443 (2001).

15. D. M. Cox, R. Brickman, K. Greegan, and A. Kaldor, Z. Phys. D, 19, 353 (1991). 